\title{
Téoros
}

Revue de recherche en tourisme

\section{Évolution du marché des touristes américains au Québec (1972-1989)}

\section{Patrick Cluzeau}

Volume 9, numéro 2, juillet 1990

Le marché américain

URI : https://id.erudit.org/iderudit/1080024ar

DOI : https://doi.org/10.7202/1080024ar

Aller au sommaire du numéro

Éditeur(s)

Université du Québec à Montréal

ISSN

0712-8657 (imprimé)

1923-2705 (numérique)

Découvrir la revue

\section{Citer cet article}

Cluzeau, P. (1990). Évolution du marché des touristes américains au Québec (1972-1989). Téoros, 9(2), 3-8. https://doi.org/10.7202/1080024ar d'utilisation que vous pouvez consulter en ligne.

https://apropos.erudit.org/fr/usagers/politique-dutilisation/ 


\section{Évolution du marché des touristes américains au Québec (1972-1989)}

L'évolution du marché des touristes ${ }^{(1)}$ américains au Québec depuis 1972 et l'analyse de ses caractéristiques seront abordées à partir de quatre indicateurs spécifiques, à savoir:

- les entrées aux frontières;

- les visites-province;

- les nuitées;

- les recettes touristiques.

Ce sont des données de l'année 1989 qui serviront de points de référence à toute l'analyse subséquente. Elles permettent de présenter ainsi le marché actuel des touristes américains au Québec:

- 1650758 entrées aux frontières;

- 2977000 visites-province;

- 10122000 nuitées;

- 669 millions de dollars de recettes touristiques pour le Québec.

\section{Les entrées aux frontières d'Américains}

\section{Origine et signification}

C'est le seul indicateur officiel disponible ${ }^{(2)}$ à l'beure actuelle qui permette de faire des comparaisons entre la performance du Québec, de 1'Ontario et du Canada sur une longue période de temps.

De fait, il permet de voir l'évolution du marché sur une période de 18 ans (de 1972, année durant laquelle il a été introduit, a 1989), ce qui n'est pas sans intérêt. Pour le Canada, il est pleinement représentatif de la réalité, les Américains devant entrer au Canada via des frontières routières, aéroportuaires ou maritimes. L'ennui provient du fait qu'il n'a pas la même valeur pour les provinces. Pour ces demières, ilne représente qu'une partie de la réalité. En prenant l'exemple du Québec, les Américains qui entrent au Quebec via les frontières situées entre les Etats-Unis et le

Patrick Cluzeau est analyete a la Direction de la recherche du ministère du Touriame du Québec.
Québec ne sont pas les seuls à venir dans la province. Il faut y ajouter les Américains quientrent vial'Ontario ou via le NouveauBrunswick mais dont la destination finale sera le Québec ou dont une partie du séjour se fera au Québec. Et ils sont nombreux. L'indicateur a done d'indéniables qualités. II a aussi ses defauts et ses limites.

En 1989, on a dénombré 1650758 entrées aux frontières de touristes provenant des États-Unis qui sont entrés au Canada via le Québec (voir la tableau 1). Ce nombre a diminué de $3 \%$ par rapport à l'année précédente, diminution moins prononcee qu'en Ontario $(-6,6 \%)$ et au Canada $(-4,4 \%)$. Cette amorce de décroissance qui se manifeste cette année au Québec et au Canada avait débuté $\mathrm{l}^{\dagger}$ an demier en Ontario. Elle fait suite à une période de croissance légère mais continue qui s'était amorcée en 1984.

\section{Tendances}

À plus long terme, des tendances peuvent être observées à l'analyse de l'évolution des entrées aux frontières entre 1972 et 1989. Dans ce cas, on constate que tout le monde a perdu du terrain, à commencer par le Canada $(-7,2 \%)$ et par l'Ontario $(-7,9 \%)$. Le Québec en a perdu nettement plus $(-21,4 \%)$ avec, comme conséquence, que la part du volume d'entréses aux frontières d'Américains au Québec par rapport au Canada, qui était de $15,3 \%$ en 1972 , n'est que de $13,5 \%$ en 1989.

L'observation et la comparaison entre deux années aussi éloignées (1972 et 1989) méritent toutefois d'être nuancées en tenant compte de toutes les années s'échelonnantentre ces deux póles. Pource qui conceme le Québec, on distingue alors trois cycles, à savoir:

- un cycle de décroissance progressive, entre 1973 et 1979 ;

- un cycle de croissance pratiquement nulle, de quasi statu quo, entre 1979 et 1983;

un cycle de reprise de la croissance depuis 1984, reprise qui s'atténue en 1988 et qui s'interrompt en 1989.
Si l'on se fie sur les intentions de voyage des Américains récemment divulguées par l'Institut canadien de recherche surle tourismedu Conference Board, un nouveau cycle de décroissance progressive aura débuté en 1989. Les chiffres traitant des entrés aux frontières lors des premiers mois de l'année 1990 tendraient aussi à le confirmer.

Le Canada, dans son ensemble, a suivi pratiquement les mêmes cycles que le Québec. Toutefois, l'Ontario a connu des diminutions nettement moins prononcées au cours des années. Il a même dépassé, en 1987, le volume d'entrées aux frontières qu'il avait en $1972(+1,8 \%)$.

Segmentation par moyen de transport

Cette évolution de l'ensemble des entrées aux frontières de touristes américains au Quebec, en Ontario et au Canada cache, cependant, des réalitís bien différentes selon les moyens de transport utilisés.

De fait, les diminutions observées entre 1972 et 1989 sont entièrement attribuables à la diminution des entrées par automobile (voir le tableau 2). Le constat fait en 1984 par Tourisme Canada ${ }^{(3)}$ pour la période 1972-1982 n'a donc pas fondamentalement changé.

Les entrées aux frontières par automobile, qui représentaient plus des trois quarts $(77,4 \%)$ de toutes les entrées aux frontières d'Americains au Québec en 1972, n'en représentent qu'un peu plus de la moitie $(58,9 \%)$ en 1989 . Elles ont diminué de $37,3 \%$. Or les voyages en automobile, à la différence des voyages d'affaires ou de congrès qui se font surtout par avion, sont surtout des voyages d'agrément ou pour visiter des parents ou des amis.

Les causes d'un tel désintéressement sont nombreuses. On y retrouve, a la fois:

- les effets de la crise énergétique qui a modifié bien des comportements de voyages de vacances;

- les effets d'une concurrence accrue venant de toutes sortes de destination, 
à commencer par les régions américaines elles-mêmes émettrices de touristes;

- les effets de l'émergence de produits touristiques nouveaux;

- les effets d'une moins grande attractivité du produit touristique québécois.

En contrepartie, les entrées aux frontières d'Américains au Québec par tous les moyens de transport autresquel'automobile ont augmenté. On aura noté, en particulier, que le volume des entrées par avion s'est substantiellement amélioré bien que l'amélioration aura été deux fois plus importante en Ontario et au Canada. II faut probablement voir là le signe d'un déplacement progressif du marché des affaires vers d'autres provinces et, en particulier, vers l'Ontario.

\section{Les visites-province d'Américains}

\section{Origine et signification}

Pour toutes les provinces, c'est l'indicateur le plus représentatif( ${ }^{(4)}$ de la réalité des voyages véritablement effectués par les Américains. Pour ce qui concerne le Québec, il prend en compte a la fois les entrées aux frontières entre les États-Unis et le Québec et les voyages qui ont débuté en Ontario, ou dans les Maritimes, mais dont une partie s'est faite au Québec. Or,en 1980, ces derniers voyages représentaient pratiquement autant $(43,3 \%)$ quelesentrées aux frontières directes. Et il n'y a pas lieu de croire que ce phénomène est différent en 1989.

Sil'indicateur "visite-province" corrige les défauts du précédent, l'ennui, dans son cas, provient du fait que c'est un indicateur que Statistique Canada a introduit en 1976 et a cessé de publier à partir de 1981 à cause du trop faible nombre de répondants àl'enquête qui rendait ses résultats peu fiables. C'est la raison pour laquelle, depuis cette date, on ne dispose d'aucune donnée provenant de Statistique Canada sur les volumes de visites-province, sur la durée du séjour, sur les dépenses et sur les caractéristiques des visites-province de touristes américains venus tant au Québec que dans les autres provinces canadiennes.

\section{Des changements attendus}

On ne peut que se réjouir de savoir que cette regrettable situation sera corrigée en 1990. Statistique Canada mènera alors, dans la

\section{TABLEAU 1}

Nombre d'entrées aux frontières de touristes des États-Unis, entrés au Canada via le Québec et l'Ontario, entre 1972 et 1989

\begin{tabular}{llll}
\hline Année & Québec & Ontario & Canada \\
\hline 1972 & 2004054 & 7126990 & 13067882 \\
1973 & 2053089 & 7410733 & 13523106 \\
1974 & 1943011 & 7262262 & 12735674 \\
1975 & 1838345 & 7063647 & 12495167 \\
1976 & 1688265 & 6492637 & 11068383 \\
1977 & 1567516 & 6535205 & 11451045 \\
1978 & 1543984 & 6442109 & 11276779 \\
1979 & 1395604 & 6334116 & 10908597 \\
1980 & 1452434 & 6347025 & 10964553 \\
1981 & 1426135 & 6364306 & 10968470 \\
1982 & 1410202 & 6064306 & 10462467 \\
1983 & 1374960 & 6234003 & 10908791 \\
1984 & 1446147 & 6504872 & 11295046 \\
1985 & 1530151 & 6630883 & 11557323 \\
1986 & 1635712 & 6895133 & 13609707 \\
1987 & 1688582 & 7216083 & 12719799 \\
1988 & 1701701 & 7073855 & 12763017 \\
1989 & 1650758 & 6606748 & 12195430
\end{tabular}

Souree: Statistique Canada, Voyages entre le Canada et les autres pays, catalogue 66001 et Cansim.

\section{TABLEAU 2}

Touristes des États-Unis entrés au Canada via le Québec et via l'Ontario selon le moyen de transport en 1972,1980 et 1989

\begin{tabular}{|c|c|c|c|c|c|c|}
\hline & & 1972 & 1980 & 1989 & $\begin{array}{c}\text { Variation } \\
1972 / 1980 \\
\delta_{0}\end{array}$ & $\begin{array}{c}\text { Variation } \\
1972 / 1989 \\
\%\end{array}$ \\
\hline Automobile & $\begin{array}{l}\text { Quebec } \\
\text { Ontario } \\
\text { Canada }\end{array}$ & $\begin{array}{r}1550690 \\
6156496 \\
11151463\end{array}$ & $\begin{array}{r}834073 \\
4885090 \\
7831214\end{array}$ & $\begin{array}{r}973022 \\
4828479 \\
8416974\end{array}$ & $\begin{array}{l}-\quad 46,2 \\
-\quad 20,7 \\
-\quad 29,8\end{array}$ & $\begin{array}{l}=37,3 \\
=21,6 \\
=24,5\end{array}$ \\
\hline Avion & $\begin{array}{l}\text { Quebec } \\
\text { Ontario } \\
\text { Canada }\end{array}$ & $\begin{array}{r}285002 \\
483135 \\
1055096\end{array}$ & $\begin{array}{r}357066 \\
808780 \\
1733775\end{array}$ & $\begin{array}{r}442947 \\
1103268 \\
2324972\end{array}$ & $\begin{array}{r}+\quad 25,3 \\
+\quad 67,4 \\
+\quad 64,3\end{array}$ & $\begin{array}{l}+55,4 \\
+128,4 \\
+120,4\end{array}$ \\
\hline Autocar & $\begin{array}{l}\text { Quebec } \\
\text { Ontario } \\
\text { Canada }\end{array}$ & $\begin{array}{l}159704 \\
381907 \\
683534\end{array}$ & $\begin{array}{l}192309 \\
403207 \\
790806\end{array}$ & $\begin{array}{l}184382 \\
348147 \\
749869\end{array}$ & $\begin{array}{r}+\quad 20,4 \\
+\quad 5,6 \\
+\quad 15,7\end{array}$ & $\begin{array}{r}15,6 \\
+\quad 8,8 \\
+\quad 9,7\end{array}$ \\
\hline Bateau & $\begin{array}{l}\text { Quebec } \\
\text { Ontario } \\
\text { Canada }\end{array}$ & $\begin{array}{r}2110 \\
102995 \\
140980\end{array}$ & $\begin{array}{r}2958 \\
121787 \\
206319\end{array}$ & $\begin{array}{r}3108 \\
168571 \\
290150\end{array}$ & $\begin{array}{l}+\quad 40,2 \\
+\quad 18,2 \\
+\quad 46,3\end{array}$ & $\begin{array}{l}+\quad 47,3 \\
+\quad 63,7 \\
+\quad 105,8\end{array}$ \\
\hline $\begin{array}{l}\text { Autres } \\
\text { moyens* }\end{array}$ & $\begin{array}{l}\text { Quebec } \\
\text { Ontario } \\
\text { Canada }\end{array}$ & $\begin{array}{r}6548 \\
2457 \\
36809\end{array}$ & $\begin{array}{r}66028 \\
128161 \\
402439\end{array}$ & $\begin{array}{r}47299 \\
158283 \\
413465\end{array}$ & $\begin{array}{r}+908,4 \\
+5116,2 \\
+993,3\end{array}$ & $\begin{array}{l}+622,3 \\
+6342,1 \\
+1023,3\end{array}$ \\
\hline $\begin{array}{l}\text { Source: Stat } \\
\text { * train, vehict }\end{array}$ & $\begin{array}{l}\text { ique } \mathrm{Ca} \\
\text { es motor }\end{array}$ & $\begin{array}{l}\text { Voyages e } \\
\text { motocyclet }\end{array}$ & $\begin{array}{l}\text { le Canada } \\
\text { piétons. }\end{array}$ & a et les autre & says, Cansim. & \\
\hline
\end{tabular}

4 TÉonos - Yat 2, no. 2, pillat 100 
foulee des recommandations du Groupe national sur les données touristiques, une enquête sur les voyages internationaux (EVI) en ayant recours à une méthodologie nouvelle permettant d'avoir un suivi des questionnaires laissés aux répondants échantillonnés. De plus, le fait que les ministères du Tourisme du Québec et de l'Ontario se soient associés financièrement à Statistique Canada afind'accroître la taille des échantillons de l'enquête permettra d'obtenirdes données beaucoup plus fiables pour chacune des deux provinces. Cela permettra ainsi de connaitre, pour la première fois sur une base annuelle, la répartitiondes flux touristiques attribuables aux visites-province d'Américains dans les principales régions touristiques du Québec de mêmeque la part de recettes touristiques qu'elles en retirent. Cela permettra aussi d'avoir des profils détaillés des différentes clientèles de touristes américains, tant des profils en termes de caractéristiques socioéconomiques qu'en termes de comportements de voyage.

Pour pallier à l'absence de données provenant de Statistique Canada concemant les visites-province d'Américains entre 1981 et 1989 , le ministère du Tourisme du Québec a fait, tous les ans, des estimations ${ }^{(5)}$ concernant le Québec. Elles sont à utiliser avec prudence bien que, se référant à la situation observée dans les entrées aux frontières, elles suivent les mêmes tendances.

\section{Tendances}

C'est ainsi que l'observation et la comparaison des visites-province d'Américains au Québecentre 1976et 1989 (voir le tableau 2) fait apparaître pratiquement les trois mêmes cycles que ce qui concerne les entrées aux frontières d'Américains au Québec, à savoir:

- un cycle de décroissance progressive, entre 1976 et 1979;

- un cycle de croissance pratiquement nulle, de quasi statu quo, entre 1980 et 1983;

- un cycle de reprise de la croissance depuis 1984, reprise qui s'atténue en 1988 et qui s 'interrompt en 1989 alors que le nombre de visites-province a diminué de $3 \%$.

La grande différence se situe dans l'année 1980 alors que, en comparaison avecl'année précédente, le volume de visites-province s'est accru beaucoup plus que celui des entrées aux frontières. La principale conséquence est que le volume de visitesprovince observé en 1980 venait, pour la première fois, de dépasser $(+4,7 \%)$ celui de 1976 alors que le volume d'entrées aux frontières en 1980 accusait toujours un deficit de $14 \%$ par rapport à 1976 . C'est donc beaucoup plus via l'Ontario et les Maritimes que directement entre les frontières USA/Québecque les Américains tendaient à venir au Québec.

\section{Origine géographique des touristes}

Ces visites-province d'Américains ont principalement pour origine quatre régions des États-Unis qui totalisent plus des trois quarts des voyages qu'ils ont effectués au Québec. Ils'agit de la Nouvelle-Angleterre $(30,9 \%)$, de l'Atlantique Centre $(25,8 \%)$, du Centre Nord-Est $(15,2 \%)$ et de 1'Atlantique Sud $(11,2 \%)$. II a déjà été démontre ${ }^{(6)} q u$ 'à l'intérieur de ces régions, certains Etats sont beaucoup plus émetteurs de touristes vers le Québec que d'autres. C'est en particulier le cas du Massachusetts dans la région de la Nouvelle-Angleterre, de l'État de New York dans la région de l'Atlantique Centre, de l'Ohioet de l'Illinois dans la région du Centre Nord-Est et de la Floride, de la Virginie et du Maryland dans la région de l'Atlantique Sud.

\section{Buts du voyage et expérience recherchee}

Plus de la moitié des visites-province d'Américains au Québec ( $54 \%)$ se font dans un but "d'agrément", les voyages "d'affaires et de congrès" en justifiant le cinquième $(21,3 \%)$ alors que les voyages pour "visiter des parents ou des amis" en justifient autant $(22,7 \%)$.

L'enquête Longwoods ${ }^{(7)}$ aura permis de mieux connaître les caractéristiques des touristes d'agrément américains ayant l'intention de venir au Québecet de montrer que les produits qu'ils veulent consommer au Québec sont, par ordre d'importance décroissante:

- l'expérience "grande ville" qui consiste à "venir y faire des achats, visiter des musées, trouver diverses attractions, aller au restaurant, assister à des pièces de theâtre et des concerts ou,tout simplement flâner,etdécouvrir les curiosités locales";

- l'expérience "circuit touristique" qui consiste "à se déplacer en automobile, en autocar ou en train dans des régions renommées pour la beauté de leur paysage ou présentant un intérêt culturel ou général";

- l'expérience "villégiature"qui consiste à séjourner "dans un centre ou une région de villégiature où une vaste gamme d'activités comme les jeux et les sports de plage, le ski, le golf et le tennis peuvent se pratiquer sur place ou à proximite";

- l'experience "plein air" dans "un endroit où la nature a été préservée en son état primitif et où l'on peut se consacrer à des activités comme le camping, la chasse, la pêche et les promenades à pied ou en radeau".

Ces caractéristiques different peu de celles mises en évidence dans l'enquête faite ${ }^{\left({ }^{()}\right.}$ auprès des touristes d'agrément de l'État de New York, du Massachusetts et de l'Ontario a partir des données recueillies lors de l'évaluation de la campagne publicitaire de l'été 1987. Elle avait toutefois permis de constater que l'importance relative de ces différentes experiences varie nettement selon l'état de résidence. Pour les résidents du Massachusetts, les voyages dans une "grande ville" sont en première place alors que les "circuits touristiques" sont en quatrième et dernière position. C'est pratiquement l'inverse pour les résidents de l'Etat de New York.

L'évolution récente des voyages des touristes américains au Québecet au Canada est à mettre en perspective avecl'évolution des voyages des touristes américains vers les principales destinations étrangères qu'ils fréquentent. On constate alors, comme le montrent les plus récentes données obtenues du U.S.Tourismand TravelAdministration (voir le tableau 4), que les destinations Mexique et Outre-Mer ont progressé beaucoup plus au cours des cinq dernières années que les destinations Québec et Canada. Entre 1988 et 1989, leurs performances ont été meilleures que celle du Canada et du Québec.

Les prévisions publiées en octobre dernier par le U.S. Travel Data Center concernant les voyages des touristes américains vers les principales destinations étrangères en 1990 affichent une progression de $4 \%$ du volume de voyages par rapport à l'année précédente. Quant à la part de marché de chacune des quatre grandes destinations des Américains, elle demeurerait la même qu'en 1989, à savoir le Mexique (33\%), le Canada $(31 \%), 1^{\prime}$ Europe $(17 \%)$ et les autres destinations Outre-Mer (19\%). Cela 
signifierait que le volume de voyages de touristes américains au Canada progresserait de $4 \%$ en 1990 par rapport a 1989. Il faut très certainement voir là un scénario particulièrement optimiste de l'évolution des voyages que des chiffres plus récemment publiés tendraient à infirmer, du moins en ce qui conceme le Québec et le Canada.

\section{Les nuitées d'Américains}

\section{Origine et signification}

C'est un indicateur particulièrement révelateur de l'importance de tout marché touristique puisqu'il tient compte a la fois du volume de voyages effectués et de la duréc du séjour des touristes. Or, comme on sait que la durée du séjour d'un touriste differe beaucoup selon son origine géographique (ainsi, par exemple, la durée moyenne de sejour d'un touriste québecois voyageant au Québec est de 2,6 nuitées alors que celle d'un touriste étranger provenant de pays autres que les États-Unis est de 8,7 nuitees), la mesure du volume de nuiteses ${ }^{(0)}$ prend toute sa signification.

\section{Des différences selon les buts du voyage}

La durée moyenne de séjour d'un touriste américain au Québec en 1989 est estimée à 3,4 nuitées, la même qu'en 1980. Cette estimationest d'autant plus plausible que la durée de séjour des touristes américains au Canada n'a pratiquement pas changé entre $1980(4,6$ nuitees) et 1988 (4,4 nuitees).

Il faut, toutefois, avoir à l'esprit que la durée de séjour differe nettement selon le but du voyage du touriste. Celui venu faire un voyage d'agrément $(3,0$ nuités) ou un voyaged'affairesou de congrès ( 3,1 nuitées) connailtune duréc de sejour moins prolongée que celui venu voir des parents ou des amis $(4,5$ nuitées) ou pour des raisons personnelles ( 4,6 nuitées). C'est une observation qui se répète au fil des ans et quise faitégalement chezles autres marchés touristiques.

Ces 10122000 nuitées de touristes américains au Quebec representent $15,9 \%$ des nuitées passées au Québec par les touristes de toutes origines géographiques confondues. Cette part relative n'a pratiquement pas change au cours des dernières années mais on constate un accroissement notable par rapport au début des années ' 80 alors que cette part relative était de I'ordre de $13 \%$. Cet accroissement
TABLEAU 3

Estimations relatives aux voyages des touristes américains hors des Etats-Unis entre 1985 et 1989

\begin{tabular}{|c|c|c|c|}
\hline Destination & $\begin{array}{l}1989 \\
000\end{array}$ & $\begin{array}{c}\text { Taux de } \\
\text { variation } \\
1989 / 1988 \\
\%\end{array}$ & $\begin{array}{c}\text { Taux de } \\
\text { Yariation } \\
1989 / 1985 \\
\%\end{array}$ \\
\hline $\begin{array}{l}\text { Canada* } \\
\text { Québec (visites-province) } \\
\text { Mexique } \\
\text { Outre-Mer, dont: } \\
\text { - Europe } \\
\text { - Caraibes } \\
\text { - Asie } \\
\text { - Amérique du Sud } \\
\text { - Amérique Centrale } \\
\text { - Océanie } \\
\text { - Moyen Orient } \\
\text { - Afrique }\end{array}$ & $\begin{array}{r}12675 \\
2977 \\
13690 \\
14925 \\
6930 \\
3735 \\
1975 \\
875 \\
635 \\
535 \\
195 \\
45\end{array}$ & $\begin{array}{l}-5,0 \\
-3,0 \\
+2,0 \\
+3,0 \\
+7,0 \\
-2,0 \\
+6,0 \\
-2,0 \\
+14,0 \\
-2,0 \\
-5,0 \\
-7,0\end{array}$ & $\begin{array}{l}+5,0 \\
+6,3 \\
+31,0 \\
+18,0 \\
+8,0 \\
+17,0 \\
+36,0 \\
+57,0 \\
+62,0 \\
+61,0 \\
-22,0 \\
-60,0\end{array}$ \\
\hline Toutes destinations confondues & 41290 & $+1,0$ & $+17,0$ \\
\hline
\end{tabular}

* Varie legèrement par rapport aux estimations de Statistique Canada.

Source: US. Tourism and Travel Administration, sauf pour le Québec, février 1990.

\section{TABLEAU 4}

Nombre de visites-province de touristes américains, venus au Québec et au Canada entre 1976 et 1989

\begin{tabular}{rrrr}
\hline Année & Québec & Ontario $^{1}$ & Canada $^{1}$ \\
\hline 1976 & 2446 & 7130 & 14258 \\
1977 & 2347 & 6818 & 14124 \\
1978 & 2341 & 6585 & 13361 \\
1979 & 2130 & 6545 & 13746 \\
1980 & 2560 & 6405 & 13735 \\
1981 & 2555 & & \\
1982 & 2438 & & \\
1983 & 2543 & & \\
1984 & 2633 & & \\
1985 & 2800 & & \\
1986 & 2968 & & \\
1987 & 3036 & & \\
1988 & 3069 & & \\
1989 & 2977 & & \\
\hline
\end{tabular}

Sources: 1-Statistique Canada, Voyages entre le Canada er les autres pays, cataloguc 66201. 2- Estimations du ministere du Tourisme.

1. Non disponible depuis 1981 pour le Canada et l'Ontario.

\section{FIGURE 1}

Part relative des nuitées des touristes au Québec, entre 1980 et 1989, selon les marchés géographiques d'origine

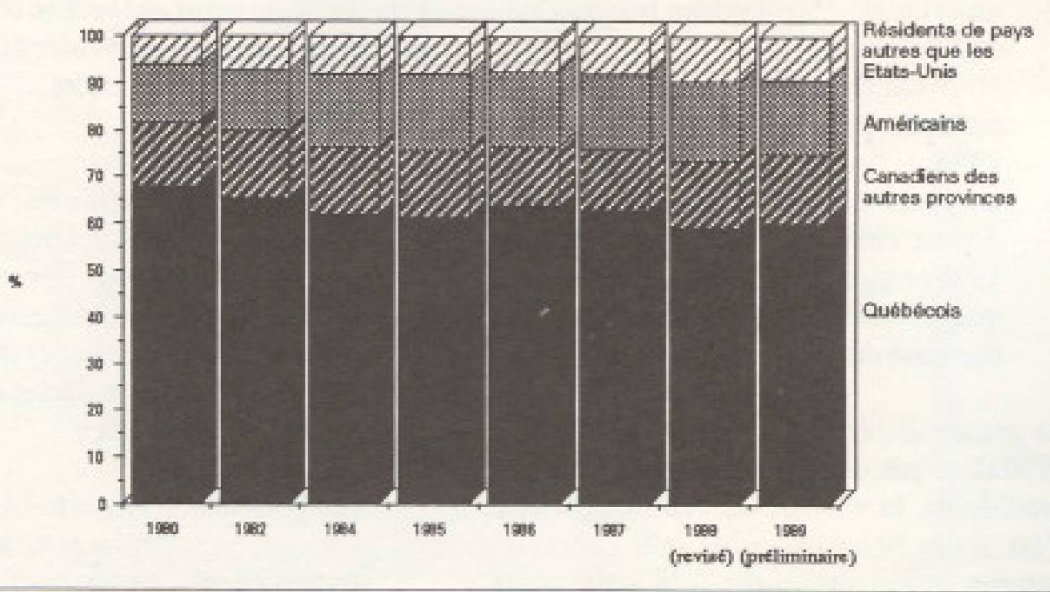




\section{FIGURE 2}

Importance relative des visites-province, des nuitées et des dépenses selon les buts de voyage des touristes américains venus au Québec, en 1989

Visites-province [2 977 oon]

Nultóes: (10 122000$)$

Dépenses 669 millions de \$)

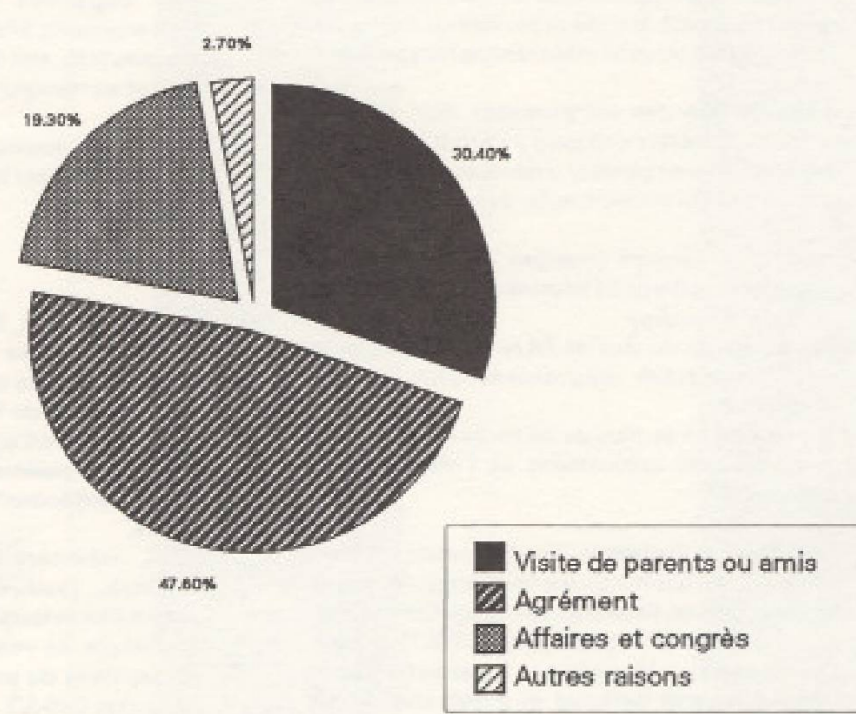

Visite de parents ou amis

Q. Agrément

Affaires et congrès

Autres raisons
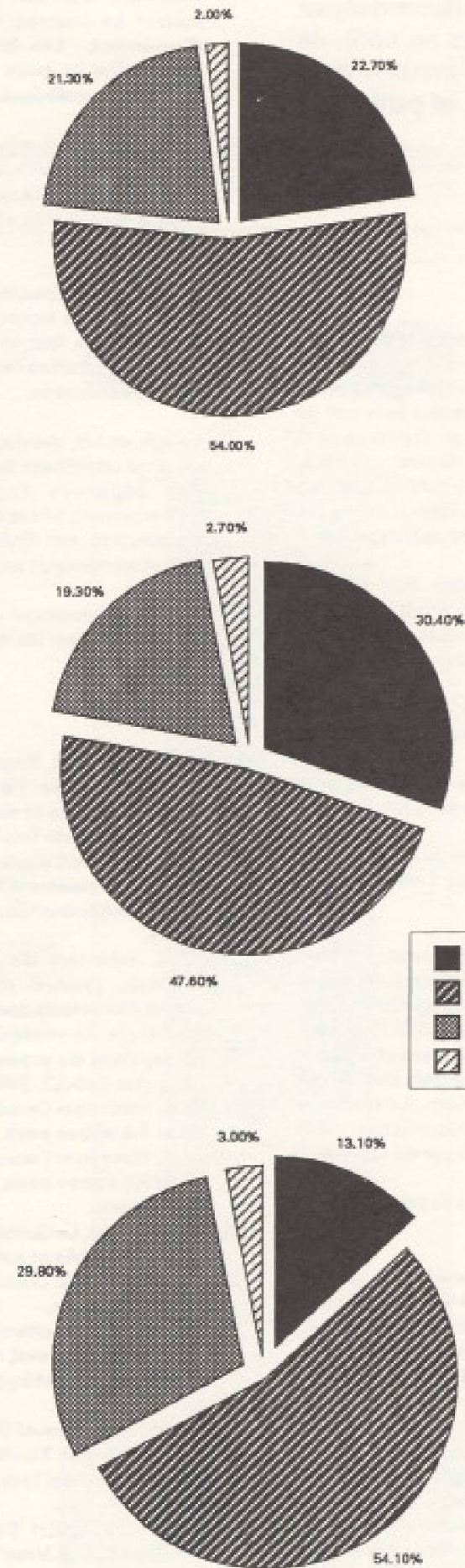

découle, en partie, de l'augmentation du volume de touristes américains au Québec depuis 1984 mais aussi, et surtout, de la diminution considérable du volume de nuitées des touristes québécois au Québec comme on peut le voir dans la figure 1 .

L'importance de ce volume de nuitées fait du marché américain le plus important marché touristique du Québec, après le marché québécois, en 1989. Il occupe également la seconde place en termes de recettes touristiques pourle Québec, comme on pourra le voir dans les lignes qui vont suivre.

\section{Les recettes touristiques découlant de la venue des touristes Américains}

\section{Origine et signification}

Elles constituent un excellent indicateur pour mesurer l'importance économique du tourisme pratiqué par les Américains au Québec. Ces recettes ${ }^{(10)}$ sont de l'ordre de 669 millions de dollars en 1989. Elles représentent la part la plus considérable des recettes touristiques du Québec au chapitre du tourisme ${ }^{(11)}$ international $(66,5 \%)$ et près du cinquieme des recettes touristiques totales du Québec $(17,7 \%)$ qui sont estimées, sur une base préliminaire, a 3,8 milliards de dollars en 1989.

Les recettes découlant de la venue de touristes américains au Québec ont nettement progressé entre 1980 et 1989 $(+109,1 \%)$ en dollars courants) mais la progression réelle a été beaucoup plus modeste ( $+22,2 \%$ en dollars constants).

Des différences selon les buts du voyage

Plus de la moitié de ces recettes $(54,1 \%)$ découlent du tourisme influençable par le marketing touristique, le tourisme d'agrément, alors que près du tiers $(29,8 \%)$ découlent du tourisme de congrès et surtout d'affaires (voir la figure 2). Le tourisme dont le but principal est de visiter des parents ou des amis, qui représente $22,7 \%$ des visites-province effectuées et $30,4 \%$ des nuitées, ne représenteque $13,1 \%$ des recettes touristiques. Cette disproportion découle du comportement même de ces derniers qui, $s$ 'hébergeant bien souvent chez des parents ou des amis, ont à la fois une durée de séjour plus prolongée que les autres touristes et une dépense quotidienne moins prononcée ( 28 \$ parnuitée-personne en 1988 
comparativement à $102 \$$ par nuitéepersonne d'affaires et de congrès et $75 \$$ par nuitée-personne d'agrément).

Cela illustre bien que chaque segment de clientèle a des comportements de voyage et de dépenses fort différents. Les touristes d'affaires et de congres sont ceux ayant le plus recours à 1 hébergement commercial, de type hôtel-motel, et au type de dépense qui en découle. Les touristes d'agrément ont recours à de $\mathrm{l}^{\prime}$ hébergement commercial plus diversifié, incluant le camping et le caravaning, et à de l'hébergement non commercial. Les touristes visitant des parents ou des amis ont surtout recours a la résidence de ces derniers. Ces comportements différents s'observent chez toutes les clientèles touristiques et non uniquement chez les touristes américains.

\section{Regard sur le compte des voyages}

Ces recettes touristiques de 669 millions de dollars provenant de la venue de touristes américains au Québecen 1989 sont àmettre en perspective avec les dépenses des touristes québécois aux États-Unis qui sont estimées, sur une base préliminaire, à 1060 milliard de dollars pour la même année. II endécoule un déficit au compte des voyages des touristes avec les États-Unis de l'ordre de 391 millions de dollars en 1989. D'une année à 1 'autre, 1 'ampleur de ce dernier a variés sous l'influence de multiples facteurs, tels le volume de voyage, l'ampleur des dépenses et le taux de change. En comparaison avec 1980 , et en dollars courants, il a pratiquement doublé.

En 1988, le déficitdu compte de voyages du Canada avecles États-Unispourles voyages d'une nuit ou plus (excluant les frais de transport pour se rendre au Canada, ceci dans le but de rendre les donnees comparables avec celles du Québec) était de 1'ordre de 954 millions de dollars et l'on estime qu'il sera plus f́levé en 1989.

\section{En conclusion}

Toutes ces donnés et toute cette analyse conduisent à une conclusion évidente. Que 1 'objectifà atteindre au cours des prochaines années soit une diminution du déficit du compte des voyages des touristes avec les États-Unis ou soit uniquement un accroissement du volume d'affaires, donc des recettes, provenant de la venue de touristes américains, le Québec, comme le Canada, devra redoubler d'efforts pour reconquérir une plus grande place dans les destinations préférées des touristes américains. Et, comme le soulignait récemment l'Institut canadien de recherche sur le tourisme, ces efforts passeront par des actions plus ciblées auprès de segments de clienteles spécifiques. Les populations noires, hispaniques et asiatiques étaient, alors, nommément mentionnées de même que les marchés du tourisme de court séjour (fins de semaine prolongées ou non), du tourisme culturel, sportif, d'aventure et du tourisme de circuits auprès, en particulier, des personnes retraitées. $f$

\section{NOTES}

(1) En conformitó avec les definitione internationalos de l'OMT et de r'OCDE, les touristes sent definis, dana le présent article, comme les peraonnes ayant fait un voyage de plus de 24 houres (une nuit ou plus) impliquant te recours al de lhobergement corrmercial ou privé. Saut indication contraire, cette definition englobe tous les buta de woyege à savoir l'egrément, la vieite de parents ou d'amis, les aftaires ou les congrès et les raisona personnelles.

Avant de faire dea comparaieena avee d'autres données, le lecteur doit avoir a l'esprit la diversité des definitions employeses, parfois, pour definir "un touriste" et qui peuvent varier de:

tous lea woyageurs (voyages de moins de 24 houres et de plus de 24 heures/ asns diatinction de la durée du voyage:

les voyageura de plus de 24 heures fune nuit ou plus) voyageant uniquement dans un but d'agrément:

les voyageura de plus de 24 heurea (une nuit ou plus) utilisant uniquement de l'hébergement commercial.

(2) Il eartutiliaépar Statistique Canada dans la publication monauelle 666001 P) intitules Tourlecope. Voyages Internatlonaux. Renseignemente proflminalres, et dana la publication trimestrib lle (66001) intitulés Tourlecope. Voyages Internatlonaux. Dénombrement national ot provineial. II eat tgalement disponible dana Cansim. Le ministdre du Touriame I'utilise dans sa publication menauelle intitulee Le barombtre tourletlque du Oubbec.

(3) Voir Le marchó ambricain dee voyages, 30 ddtion.

(4) Il a été utiliás par Statistique Canada dans a publication annuelle (66 201) intitulés Voyages entro lo Canada et les autres pays entre 1976 et 1980 en ce qui conceme les Amóricsins. Avant 1976 , I'indicateur utiliaé était celui de la "province de destination" qui ne tenait pas compte de la partie du voyage faite en traversant une province si ce n'ótait pas la destination finale du voyege. C'est la raisen pour laquelle les donn bee publíeesà pertir de 1976 ne peuvent pas se comparer aux donnees publiées antérieurement. Les rdsultate de l'enquête utilisant le concept de viaite-province ne sont publiés qu'une fois par année, contrairement aux entrees aux frontieres qui sont publides sur une base mensuelle.

(5) Voir Le Qubbec touristique. Indleateurs sur les marchós et sur less secteurs touriktiques de 1980 à 1988.
(6) Voir Le Cuébec tourlstique, Indleateurs sur les marchbe ot sur les secteurs tourlatlques de 1980 a) 1988, pages 104 a 107. Le poids de chaque Etat est alors fourni en termes de visites-province, de nuitśbs at de dépenenes.

(7) Les rósultats concemant apécifiquement le Quóbec ont fait lobjet d'une analyee et d'une publication diatincte faite conjointement par le ministere du Touriame du Quóbec at le ministère de l'Expansion industrielle régionale du Canada. Ello porte le titre suivant Le marchb amb́ricain des voyages d'agróment. Les Intentlons de voyage des Amerieains of leurs perceptions du pro-dult touristique quobbécois.

(6) Mâme si, compte tenu des critores de aélection des répondante de lenquête, elle ne prúsenté pas une image complète des touriates d'agrément des régions considérébas, alle n'en demeure pas moins: tràa indicative.

(9) If s'agit de la nuit pasede per un touriate en voyage. quel que soit le moyen d'hébergement utilisb, Si deux touristes font un voyage pendant lequel ils pessont trois nuits à l'extérieur de leur domicile, on compte aix nuitées.

(10) Il s'agit, en tait, des dépenaes des touriates amerí cains qui constituent des recettes pour le Quebec. Ces dépenses englobent celles libes à l'hẻbergement, al lalimentation (ce qui comprend la restauration), aux divartiasements, aux achats divera et au transport au Quóbec.

(11) II s'sgit du touriame pratiques a la fois par lea Américaine et par les résidents de pays autres que les Ettats-Unis.

\section{BABUOGRAPHIE}

BOUCHARD, Gabriel, Enquôts aupros de tourletes d'agróment de l'État de Now York, du Massachussetts et de P'Ontarlo, d́tó 1987, Québec, ministiłe du Tourisme, 1988, 89 peges.

CANADA, Tourieme Canada, Lo marché ambirkeain des voyages, troiaieme fdition, Ottawa, riniatère de l'Expansion économique régionale, juillet 1984, 93 pages.

CANADA, miniatère de l'Expansion économique régionale, Québeo, miniatère du Touriame, Lo march $\phi$ a mórieain dee voyages d's gróment. Les intentions de voyage des Amb́rlealne ot lours perceptions du prodult tourlatkque qub́b6cels, catalogue C29-3/2, 249 pagea.

CANADA Statistique Canada, Voyages entre le Canada ot les autres pays, Cansim.

CANADA, Statiatique Cenada, Voyagee entro le Canada et les autres pays, 1976 a 1980, catalogue 66 201, Ottawa.

CLUZEAU, Petrick, Le Qubbect touristique. Indicateurs sur los marches ot aur les secteurs tourlatiques. de 1980 a 1988, Québec, ministère du Touriamo. 1989, 619 paged.

ETATS-UNIS, U.S.Touriam and Travel Administration, U.S. Outbound Travel, 4th Annual Travel Reviow Conference. Washington, fevrier 1990, non pagine.

ETATS-UNIS, U.S. Travel Data Center, 1990 Outlook for Travel and Tourism. Proceedings of the Fifteenth Annual Travel Outlook Forum, octobre 1989, 127 pages.

INSTITUT CANADIEN DE RECHERCHE SUR LE TOURISME, U.S. Vacation TravelOutlook, Conference Board du Cansda, Ottawa, hiver 1990, non paginá.

SOCIETE QUEBBECOISE DE PROMOTION TOURISTIQUE, Profil de marehe. Etats-Unis, Montráal, ministère du Touriame, novembre 1988, non pagine. 